\title{
Clinical Reasoning: Refractory status epilepticus in a primigravida
}

Hang Shi, MD, Genna Waldman, MD, Steven Tobochnik, MD, Sheng-Han Kuo, MD, and Alison Pack, MD

Neurology ${ }^{\circledR}$ 2019;92:968-972. doi:10.1212/WNL.0000000000007507
Correspondence

Dr. Shi

hs2959@cumc.columbia.edu

\section{Section 1}

A 29-year-old woman, gravida 1 para 0, 14 weeks pregnant, presented with first seizure of life. Thirty minutes before the seizure, she reported a new mild bifrontal headache and visual changes described as difficulty seeing her right fingers when using her phone. She then had witnessed right arm extension, followed by shaking in bilateral arms and legs for 2 minutes, and bladder incontinence followed by 30 minutes of postictal confusion. Her medical history was notable for ataxia, myoclonus, and foot numbness diagnosed 4 years prior as sensory ataxic neuropathy, dysarthria, and ophthalmoplegia (SANDO) syndrome; whole exome sequencing had confirmed compound heterozygous polymerase gamma ( $P O L G)$ mutations. Her home medications were amantadine $100 \mathrm{mg}$ BID, coenzyme Q10 $100 \mathrm{mg} \mathrm{BID}$, and prenatal multivitamin daily.

On assessment, the patient's vital signs were normal and neurologic examination revealed a new right homonymous hemianopia. Her prior baseline examination included normal mental status, end-gaze nystagmus in all directions, mild dysarthria, intermittent myoclonic jerks, dysmetria in all extremities, decreased sensation below the knees, and wide-based gait.

\section{Questions for consideration:}

1. What is the expected localization of the patient's acute presentation?

2. What is the differential diagnosis?

\section{GO TO SECTION 2}




\section{Section 2}

Right homonymous hemianopia localizes to a left-sided postchiasmatic lesion, most likely in the occipital lobe. The semiology of right arm extension is suggestive of a focus from the left motor cortex, which could be the primary focus or propagation. Tonic posturing may also observed in seizures involving the supplementary motor area or temporal lobe.

There is a broad differential for headache, visual changes, and seizures in pregnancy. Eclampsia is considered when a pregnant woman has a seizure, but generally occurs after 20 weeks gestation with associated hypertension or proteinuria. ${ }^{1}$ Vascular etiologies include posterior reversible encephalopathy syndrome (PRES), reversible cerebral vasoconstriction syndrome, ischemic/hemorrhagic stroke, subarachnoid hemorrhage, and dural venous sinus thrombosis. Headache and seizure raise concern for encephalitis from infectious, autoimmune, or paraneoplastic processes, although less likely in the absence of altered mental status. Mitochondrial diseases including POLG-spectrum diseases are known to cause epilepsy. ${ }^{2}$ Pituitary apoplexy should be considered in a pregnant patient with new-onset headache and visual changes, although seizure is rare.

Our patient underwent MRI/magnetic resonance angiography/magnetic resonance venography brain without contrast, revealing restricted diffusion in the left occipital cortex with corresponding T2/fluid-attenuated inversion recovery (FLAIR) hyperintensity and minimal edema (figure, A). Given new seizure with a radiographic lesion, levetiracetam $750 \mathrm{mg}$ BID was started. A lumbar puncture was

Figure Brain MRI, ictal EEG, and quantitative EEG

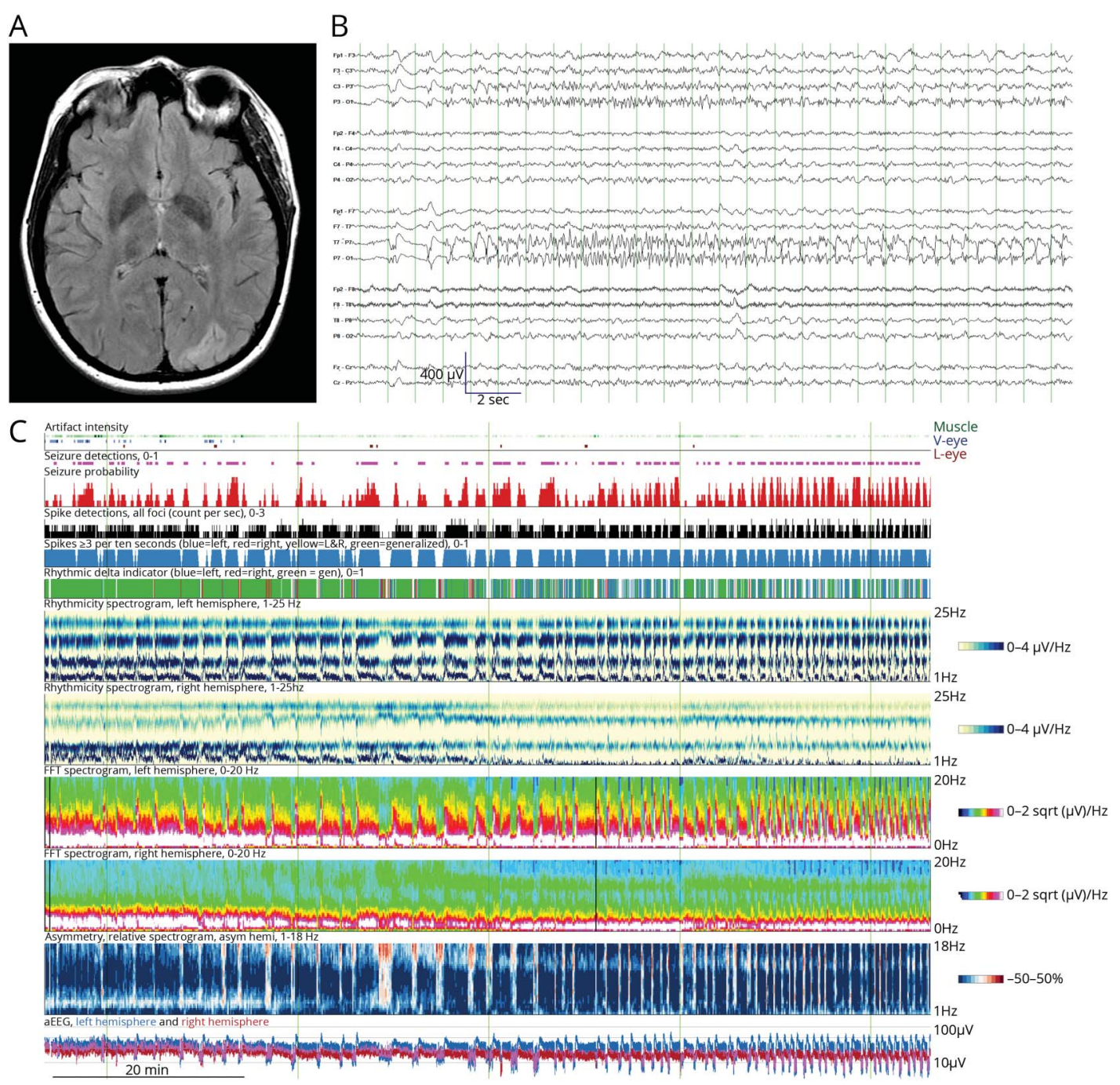

(A) Axial fluid-attenuated inversion recovery MRI demonstrates abnormal hyperintensity of the left occipital cortical convexity. (B) Representative electrographic seizure with left parieto-occipital onset. (C) Quantitative trend analysis using Persyst 13 (Persyst Development Corp., Solana Beach, CA) with artifact reduction over a continuous 93-minutes EEG segment reveals increasingly frequent seizure detections (panel 2, pink; panel 3, red), strongly lateralized to the left hemisphere (fast-Fourier transform [FFT] spectrogram panel 9 vs 10; amplitude-integrated EEG [aEEG] panel 12). Rhythmicity spectrogram of the left hemisphere (panel 7) demonstrates early peaks in high alpha and beta power followed by increase in delta power, with stereotyped seizure morphology. 
performed with opening pressure of $13 \mathrm{~cm} \mathrm{H}_{2} \mathrm{O}$ and noninflammatory CSF.

The next day, the patient developed difficulty following commands and no blink to threat bilaterally. Emergent MRI showed new diffusion restriction in the left parietotemporal cortex and right occipital and left frontal lobe, without respecting vascular territories. She had another seizure manifesting as right head version, rightward eye deviation, and bilateral tonic-clonic arm and leg movements lasting 3 minutes followed by prolonged postictal confusion. Despite IV lorazepam $2 \mathrm{mg}$ and IV levetiracetam 2,000 mg, she remained altered and had 2 more seizures of similar semiology each lasting 1 minute. She met criteria for status epilepticus (SE) with 2 seizures without return to baseline in between, and was transferred to the neurologic intensive care unit, where continuous EEG revealed nonconvulsive electrographic SE originating from the left occipital lobe (figure, B).

\section{Questions for consideration:}

1. What is the etiology of the patient's SE?

2. How does pregnancy and mitochondrial disease affect the treatment of the patient's SE?

GO TO SECTION 3 


\section{Section 3}

Based on the patient's known POLG mutation, rapidly evolving occipital predominant MRI signal changes, and focal SE, she was diagnosed with POLG-related epilepsy. This is a mitochondrial disorder with broad nervous system manifestations and is known to cause occipital lobe seizures, focal motor seizures, and SE. ${ }^{3}$ MRI brain typically shows $\mathrm{T} 2 /$ FLAIR hyperintensities, sometimes with restricted diffusion, in the occipital cortex, although other areas of cortex, thalami, and cerebellum have been reported. ${ }^{3,4}$ PRES had also been considered, but in the absence of risk factors for PRES and MRI showing cortical restricted diffusion rather than predominantly white matter abnormalities, this was less likely. In POLG-related epilepsy, EEG typically shows ictal and interictal activity originating from the occipital lobes, ${ }^{3}$ and can demonstrate posterior rhythmic high-amplitude delta with superimposed polyspikes (RHADS), which is pathognomonic for Alpers syndrome (POLG-related childhood progressive encephalopathy with intractable epilepsy and liver failure). ${ }^{4}$ By International League Against Epilepsy classification, she had acute symptomatic progressive SE due to POLG; her EEG showed cyclic left occipital seizures with left parietal-occipital RHADS (figure, C).

The treatment of POLG-related SE in pregnancy is not welldescribed. In general, SE in pregnancy should be treated with antiepileptic drugs (AEDs) and, if necessary, intubation and continuous infusion therapy, while addressing underlying causes of seizures. ${ }^{1}$ It is important to prioritize treating SE to improve maternal and fetal outcomes. ${ }^{1}$ Multiple AEDs are often required to control seizures in POLG-related epilepsy. ${ }^{2}$ There are limited data on which AEDs are recommended, although newer AEDs such as levetiracetam, lacosamide, lamotrigine, and clobazam may be less sedating and therefore afford better quality of life. ${ }^{2}$ In pregnancy, it is important to consider the potential adverse effects of AED therapy on the developing fetus. Our patient was beyond the first trimester and therefore beyond the period of highest concern for teratogenicity, but AEDs in later trimesters can still affect cognitive development. Valproate should be avoided due to risk of mitochondrial toxicity, which can manifest as hepatotoxicity, leading to liver failure and death, ${ }^{3}$ and in utero exposure has negative effects on neural tube, craniofacial, cardiac, skeletal, and cognitive development. ${ }^{5}$ IV magnesium has been effective in refractory POLG-related SE in 2 cases. ${ }^{6}$ Pulse-dose steroids, L-arginine, and supplements used in the treatment of other mitochondrial disorders are of unclear efficacy in POLG-related SE. There are reports of ketogenic diet, low glycemic index diet, and transcranial direct current stimulation in treating POLG-related epilepsy with variable improvement in seizure frequency. ${ }^{7}$

Our patient's SE was difficult to control and required continuous IV midazolam and ketamine, as well as lacosamide, levetiracetam, phenytoin, and clobazam. She was gradually weaned off midazolam and ketamine. Targeted therapy included continuous IV magnesium for 22 days, IV methylprednisolone 1,000 mg daily for 3 days, IV L-arginine $30 \mathrm{mg}$ daily, levocarnitine $990 \mathrm{mg} \mathrm{BID}$, and pyridoxine $25 \mathrm{mg}$ daily. When AEDs were weaned, frequent seizures returned, resulting in continuation of all 4 oral AEDs through discharge.

\section{Discussion}

$P O L G$ gene encodes DNA polymerase gamma, an enzyme crucial to mitochondrial DNA replication. ${ }^{7}$ Mutations in POLG are common causes of mitochondrial disease. Autosomal dominant and autosomal recessive patterns of inheritance have been described, with a wide array of symptoms presenting from infancy to adulthood. Possible manifestations include hypotonia, developmental delay, myoclonus, epilepsy, migraines, stroke-like episodes, neuropathies, ataxia, psychiatric illnesses, diabetes, liver dysfunction, and cardiomyopathy. ${ }^{2}$ Among the most severe disorders are POLG-related SE and Alpers syndrome, which often start in childhood but can present in adulthood. ${ }^{7}$ Other syndromes include SANDO, with which this patient had been diagnosed previously, as well as myoclonic epilepsy myopathy sensory ataxia, ataxia neuropathy spectrum, and progressive external ophthalmoplegia.

POLG-related epilepsy is thought to result from excessive energy depletion in metabolically active neurons with dysfunctional mitochondria. ${ }^{3,7}$ Alterations in neuronal excitability and network inhibition can in turn lower the seizure threshold. This process results in a cycle of further metabolic demands within the cortical parenchyma, thus causing injury to susceptible structures and further seizures. MRI shows typical cortical changes due to these metabolic processes and seizure epiphenomena, which are both generally reversible although atrophy may occur over time. ${ }^{3,4}$

In the absence of prior seizures, family history, or epilepsy risk factors aside from POLG mutation, it is unknown why our patient developed refractory SE at 14 weeks gestation. Her being in the second trimester could have increased her total body metabolic demands and increased vulnerability of her cortical neurons, thus provoking seizures. To our knowledge, there is not a well-characterized association between mitochondrial disorders and the development of new seizures during pregnancy. ${ }^{8}$ However, her specific compound heterozygous mutations, a paternally inherited missense variant (p.Arg627Gln) and 2 maternally inherited missense variants present in cis (p.Gly11Asp; Arg852Cys), likely heightened her risk for epilepsy since the maternal variants have been described in patients with epilepsy, and compound heterozygous mutations are associated with more severe epilepsy and shorter survival than homozygous mutations. ${ }^{7,9}$ Given the theoretical risk of increasing metabolic demand as pregnancy continues, extensive conversations were held with the patient, family, and obstetrics team with regards to continuing the pregnancy. In the absence of evidence as to how to proceed, discussions focused on the risk of further seizures and exposure to multiple 
AEDs on fetal development, as well as uncertain prognosis if she were to remain pregnant. Ultimately the patient chose to have an elective abortion at 16 weeks.

On outpatient follow-up 3 months later, despite continuing 4 AEDs, the patient had one possible seizure described as transient right visual field loss. Otherwise her neurologic examination returned to her prior baseline. Repeat MRI brain showed interval resolution of T2/FLAIR hyperintensities. She elected to start oral contraception to prevent future pregnancy.

\section{Author contributions}

H. Shi: primary patient care, drafting of manuscript, critical revision of the manuscript, literature search. G. Waldman: primary patient care, critical revision of the manuscript, literature search. S. Tobochnik: critical revision of the manuscript, literature search. S.-H. Kuo: primary patient care, critical review of the manuscript. A. Pack: critical review of the manuscript, literature search.

\section{Study funding}

No targeted funding reported.

\section{Disclosure}

The authors report no disclosures relevant to the manuscript. Go to Neurology.org/N for full disclosures.

\section{References}

1. Rajiv KR, Radhakrishnan A. Status epilepticus in pregnancy: etiology, management, and clinical outcomes. Epilepsy Behav 2017;76:114-119.

2. Cohen BH, Chinnery PF, Copeland WC. POLG-related disorders. 2010 [Updated 2018 Mar 1]. In: Adam MP, Ardinger HH, Pagon RA, et al, eds. GeneReviews [Internet]. Seattle: University of Washington; 1993-2018. Available at: ncbi.nlm.nih. gov/books/NBK26471/. Accessed November 6, 2018.

3. Janssen W, Quaegebeur A, Van Goethem G, et al. The spectrum of epilepsy caused by POLG mutations. Acta Neurol 2016;116:17-25.

4. Wolf NI, Rahman S, Schmitt B, et al. Status epilepticus in children with Alpers disease caused by POLG1 mutations: EEG and MRI features. Epilepsia 2009;50: 1596-1607.

5. Ornoy A. Valproic acid in pregnancy: how much are we endangering the embryo and fetus? Reprod Toxicol 2009;28:1-10

6. Visser NA, Braun KP, Leijten FS, van Nieuwenhuizen O, Wokke JH, van den Bergh WM. Magnesium treatment for patients with refractory status epilepticus due to POLG1-mutations. J Neurol 2011;258:218-222.

7. Anagnostou ME, Ng YS, Taylor RW, McFarland R. Epilepsy due to mutations in the mitochondrial polymerase gamma $(P O L G)$ gene: a clinical and molecular genetic review. Epilepsia 2016;57:1531-1545.

8. Karaa A, Elsharkawi I, Clapp MA, Balcells C. Effects of mitochondrial disease/ dysfunction on pregnancy: a retrospective study. Mitochondrion 2018. Epub $2018 \mathrm{Jul}$ 7.

9. Ashley N, O'Rourke A, Smith C, et al. Depletion of mitochondrial DNA in fibroblast cultures from patients with POLG1 mutations is a consequence of catalytic mutations. Hum Mol Genet 2008;17:2496-2506.

\section{New AAN Practice Management Webinar Format Digs Deeper}

The new format offers a live, 30- to 45-minute webinar with expert faculty followed by several shorter recorded online lectures that explore the topic in greater depth. Each topic concludes with a 30-minute live webchat for further participant Q\&A. Purchase a single webinar series for \$99 or purchase a 2019 Practice Management Webinar subscription for only \$189-that's less than $\$ 32$ per webinar!

\section{Webinar Series}

- January 15 - Boss, MD: Managing a Better Practice

- March 12 - Understanding How You Get Paid

- May 21 - Everything You Wanted to Know About Your Patients but Were Afraid to Ask: Having Difficult Conversations with Patients from Vulnerable Populations

- August 13 - Increasing Revenue in Your Practice: Care Models, Ancillary Services, and Other Strategies

- October 1 - Using Technology for Better Practice Management of Stroke

- November 19 - Seeing the Future Clearly: How to Succeed in 2020

Learn more and register at AAN.com/view/webinar. 


\section{Neurology}

\section{Clinical Reasoning: Refractory status epilepticus in a primigravida}

Hang Shi, Genna Waldman, Steven Tobochnik, et al.

Neurology 2019;92;968-972

DOI 10.1212/WNL.0000000000007507

\section{This information is current as of May 13, 2019}

\section{Updated Information \&} Services

References

Subspecialty Collections

Permissions \& Licensing

Reprints including high resolution figures, can be found at: http://n.neurology.org/content/92/20/968.full

This article cites 7 articles, 0 of which you can access for free at: http://n.neurology.org/content/92/20/968.full\#ref-list-1

This article, along with others on similar topics, appears in the following collection(s):

Mitochondrial disorders

http://n.neurology.org/cgi/collection/mitochondrial_disorders Status epilepticus

http://n.neurology.org/cgi/collection/status_epilepticus

Information about reproducing this article in parts (figures,tables) or in its entirety can be found online at:

http://www.neurology.org/about/about_the_journal\#permissions

Information about ordering reprints can be found online:

http://n.neurology.org/subscribers/advertise

Neurology ${ }^{\circledR}$ is the official journal of the American Academy of Neurology. Published continuously since 1951, it is now a weekly with 48 issues per year. Copyright (O 2019 American Academy of Neurology. All rights reserved. Print ISSN: 0028-3878. Online ISSN: 1526-632X.

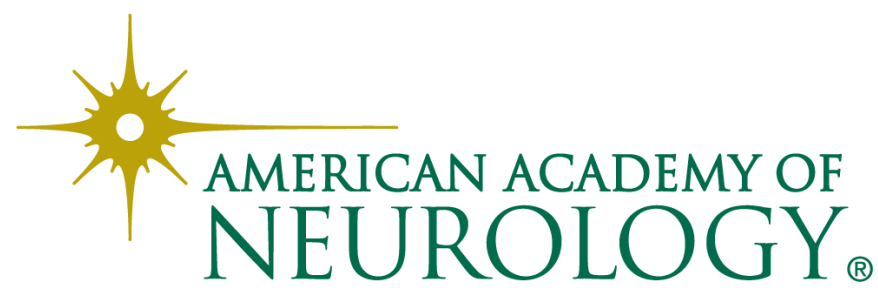

